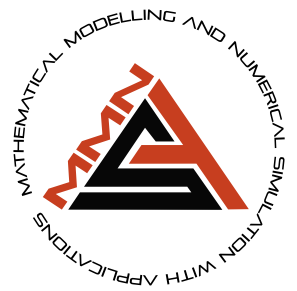

\title{
Stability analysis of an incommensurate fractional-order SIR model
}

\author{
Bahatdin Daşbaşı $1, *, \dagger$ \\ ${ }^{1}$ Kayseri University, Faculty of Engineering, Architecture and Design, Department of Engineering Basic \\ Sciences, 38039, Kayseri, Turkey \\ * Corresponding Author \\ †bdasbasi@kayseri.edu.tr (Bahatdin Daşbaşı)
}

\begin{abstract}
In this paper, a fractional-order generalization of the susceptible-infected-recovered (SIR) epidemic model for predicting the spread of an infectious disease is presented. Also, an incommensurate fractional-order differential equations system involving the Caputo meaning fractional derivative is used. The equilibria are calculated and their stability conditions are investigated. Finally, numerical simulations are presented to illustrate the obtained theoretical results.
\end{abstract}

Key words: SIR mathematical model; incommensurate order differential equation; fractional-derivative; stability analysis AMS 2020 Classification: 34A08; 34D20; 34K60; 92C50; 92D30

\section{Introduction}

The topic of fractional calculus (FC) has gained considerable popularity and importance in the last three decades, mainly because of its wide variety of applications in science and engineering. Also, it has been found that many systems can be described with fractional differential equations in many interdisciplinary fields [1]. Fractional-order differential equation (FODE) models have advantages over classical ordinary differential equation (ODE) and/or delayed differential equation models because integer derivatives are used to obtain information about only local properties of a state, while fractional derivatives describe the entire space. In other words, in FODE models, the next precise location for a physical phenomenon depends not only on the current situation, but also on all historical situations. Thus, these models not only give more realistic biological models involving memory but also expand the stability region of states [2]. Fractional-order systems (FOSs) are can be considered in two parts, as commensurate FOS (CFOS) and incommensurate FOS (IFOS) according to the derivative orders in the system. CFOS can be considered as a special case of derivative orders in IFOS [3]. Given the fact that the stability theorem of fractional differential equations favors stability analysis and controller synthesis, this motivates us to adopt stability criteria for the field of incommensurate fractional-order nonlinear systems and give sufficient conditions for determining stability [4]. Therefore, modeling of biological dynamics with IFOS is more comprehensive in terms of predicting the behavior of the system [5]. Furthermore, theorems of existence, uniqueness and dependence upon initial conditions according to some special conditions of IFOS are given in [6,7].

There are many recent studies in the literature on the stability of IFOS $[8,9,10,11]$. In addition, modeling and stability analysis of biological systems by IFOS has been frequently discussed in the literature recently $[12,13,5,7,14]$ and CFOS $[15,16,17,18,19$, $20,21,22,23,24]$

In the field of epidemiology, many schemes have been developed to mathematically model various infectious epidemics. Compartment models such as SIR modeling, which divide communities into certain main classes, are the most widely used models. The interactions between these classes are mainly determined by certain pre-mathematical formulas. The classical SIR epidemiological model was first introduced by Kermack and McKendrick in 1927. This ordinary differential equation system (ODES) models the

> Received: 17.09.2021 > Revised: 28.09.2021 > Accepted: 29.09.2021 > Published: 30.09.2021 
spread of an epidemic in a population. More recently, there has been increased interest in extending SIR models through the inclusion of fractional derivatives [27]. Modified SIR mathematical modeling through CFOS are in recent years analyzed in [28, 29, 30]. In here, the time-dependent changes in sizes of susceptible, infected and recovered individuals in a population in case of an infectious disease were investigated by mathematically modeling with IFOS. An innovation has been presented to the literature in terms of the use of IFOS in the model. In addition, the results were supported by numerical studies.

The remainder of the article is arranged as follows:

- In Section 2, the existence of equilibrium points of the proposed model and their stabilities are analyzed.

- In Section 3, the mathematical formulation of the proposed SIR model is presented. Furthermore, the threshold parameter is presented.

- Section 4 proposes the stability conditions of the mentioned biological system.

- Section 5 backs up the qualitative analysis results of the proposed IFOS. In this respect, numerical simulations are performed.

- The article ends in Section 6 with some concluding remarks.

\section{Preliminaries and definitions}

In here, it is given some basic definitions and notations with respect to follows: FODE with Caputo derivatives and locally asymptotically stability (LAS) of the equilibrium point of an n-dimensional FOS, respectively.

Definition 1 According to the definition of Caputo sense, the fractional derivative of the function $f(t)$ is defined as

$$
{ }^{C} D_{t}^{\alpha}(f(t))=\frac{1}{\Gamma(n-\alpha)}\left(\frac{d}{d t}\right)^{n} \int_{a}^{t}(t-x)^{n-\alpha-1}\left(\frac{d}{d x}\right)^{n} f(x) d x, n-1<\alpha \leq n,
$$

where $\Gamma($.$) is the Gamma function, which is described by \Gamma(x)=\int_{0}^{\infty} t^{x-1} e^{-t} d t, f:(0,+\infty) \rightarrow \mathbb{R}$ and $\alpha>0$ [31].

The Caputo fractional order sense is used in this study.

Remark 1 The nonlinear FOS can be defined as following

$$
\frac{d^{\bar{\alpha}} X(t)}{d t^{\bar{\alpha}}}=F(t, X(t))
$$

where it is considered initial conditions by $X(0)=X_{0}$, the state vectors by $X(t)=\left[x_{1}(t), x_{2}(t), \ldots, x_{n}(t)\right]^{T} \in \mathbb{R}^{n}$, the functions by $F=\left[f_{1}, f_{2}, \ldots, f_{n}\right]^{T} \in \mathbb{R}^{n}, f_{i}:[0,+\infty) x \mathbb{R}^{n} \rightarrow \mathbb{R},(i=1,2, \ldots, n)$ and the derivative-orders by $\bar{\alpha}=\left[\alpha_{1}, \alpha_{2}, \ldots, \alpha_{n}\right]^{T}$ such that $\frac{d^{\bar{\alpha}} X(t)}{d t^{\bar{\alpha}}}=\left[\frac{d^{\alpha} 1 x_{1}(t)}{d t^{\alpha_{1}}}, \frac{d^{\alpha} 2 x_{2}(t)}{d t^{\alpha}{ }_{2}}, \ldots, \frac{d^{\alpha} x_{n}(t)}{d t^{\alpha} n}\right]^{T}[32]$.

For the rest of the article, $\alpha_{i}$ is in $(0,1)$.

Definition 2 For system (2), autonomous IFOS can be presented as

$$
\frac{d^{\bar{\alpha}} X(t)}{d t^{\bar{\alpha}}}=F(X(t)), X(0)=X_{0} .
$$

Also, the equilibrium point of system (3) is found from $F(\bar{X})=0$ for $\bar{X}=\left(\overline{x_{1}}, \overline{x_{2}}, \ldots, \overline{x_{n}}\right)[6]$.

Lemma 1 Eigenvalues $\lambda_{i}$ for $i=1,2, \ldots, m\left(\alpha_{1}+\alpha_{2}+\cdots+\alpha_{n}\right)$ of system (3) are obtained from

$$
\operatorname{det}\left(\operatorname{diag}\left(\lambda^{m \alpha_{1}}, \lambda^{m \alpha_{2}}, \ldots, \lambda^{m \alpha_{n}}\right)-J(\bar{X})\right)=0,
$$

where $m$ is the smallest of the common multiples of the denominators of rational numbers $\alpha_{1}, \alpha_{2}, \ldots, \alpha_{n}$ and $J(\bar{X})=\left.\frac{\partial F}{\partial X}\right|_{X=\bar{X}}$. If all eigenvalues $\lambda_{i}$ obtained from equation (4) satisfy

$$
\left|\arg \left(\lambda_{i}\right)\right|>\frac{\pi}{2 m}
$$

then $\bar{X}$ is LAS for system (3) [33, 34].

As a result, Figure 1 shows the stability conditions of the incommensurate order SIR model given in (3), where $\alpha_{1} \neq \alpha_{2} \neq \cdots \neq \alpha_{n}<1$ and $\lambda_{i}$ for $i=1,2, \ldots, m\left(\alpha_{1}+\alpha_{2}+\cdots+\alpha_{n}\right)$. 


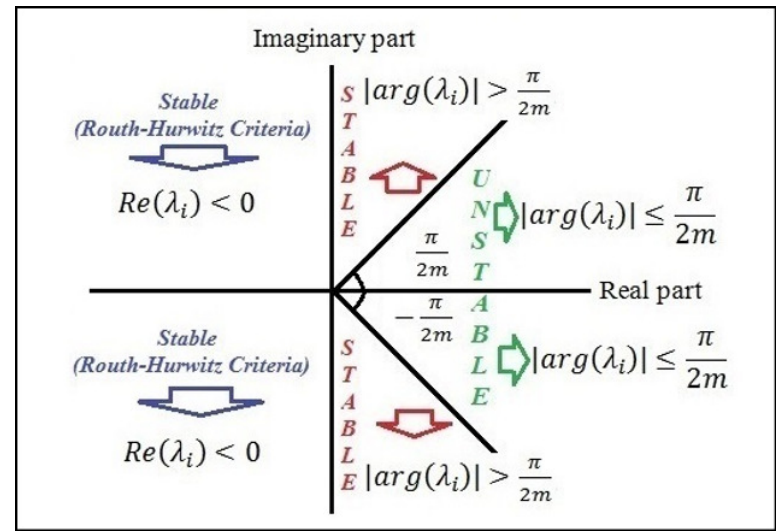

Figure 1. Regions of stability and instability of the equilibrium point in terms of the roots of the characteristic equation of the system (3) [5].

\section{The SIR model through IFOS}

We consider a SIR epidemic disease model. Define the following dependent-time $t$ :

Table 1. State variables and their meanings

\begin{tabular}{|l|l|}
\hline State Variable & Meaning \\
\hline$S(t)$ & The susceptible individuals at the $t$-time \\
\hline$I(t)$ & The infected symptomatic individuals at the $t$-time \\
\hline$R(t)$ & The recovered individuals at the $t$-time \\
\hline
\end{tabular}

Therefore, the dynamics is governed by a system of three FODE as follows:

$$
\begin{aligned}
\frac{d^{\alpha_{1}} S(t)}{d t^{\alpha_{1}}} & =\Lambda+\nu R-\eta I S-(\mu+b) S, \\
\frac{d^{\alpha_{2}} I(t)}{d t^{\alpha_{2}}} & =\eta I S-(\gamma+d+b) I, \\
\frac{d^{\alpha_{3} R}(t)}{d t^{\alpha_{3}}} & =\mu S+\gamma I-(\nu+b) R,
\end{aligned}
$$

where $t \geq 0, \alpha_{i} \in(0,1]$ for $i=1,2,3$. Also, the initial conditions are $S\left(t_{0}\right)=S_{0}>0, I\left(t_{0}\right)=I_{0}>0$ and $R\left(t_{0}\right)=R_{0}>0$ for $t>t_{0}$. Restrictions are imposed on the parameters to ensure that solutions are nonnegative. Therefore, the following conditions hold

$$
\wedge, v, \eta, \mu, b, \gamma, d>0 \text {. }
$$

In Table 2, it is illustrated parameters with their meaning.

Table 2. Parameters and their meanings in the proposed model

\begin{tabular}{|l|l|}
\hline Parameter & Meaning \\
\hline$\Lambda$ & The constant birth number in the overall population \\
\hline $\mathrm{V}$ & The immunity loss rate of recovered individuals \\
\hline$\eta$ & $\begin{array}{l}\text { The contact number, the average number of successful contacts resulting } \\
\text { in infection and made by one infected individual }\end{array}$ \\
\hline$\mu$ & $\begin{array}{l}\text { Rate of the vaccinated susceptible individuals } \\
\text { the overall population. }\end{array}$ \\
\hline$b$ & Recovery rate of the infected individual \\
\hline$\gamma$ & Average fatality rate of the infected individual due to infectious disease \\
\hline$d$ &
\end{tabular}

Therefore, Figure 2 is obtained from system (6).

Definition 3 The baseline reproduction number, often denoted as $\mathcal{R}_{0}$, describes the average number of secondary infections caused by an infected individual in a fully susceptible population. This number indicates whether the infection will spread to the population or not [35].

For the proposed model, it is described this parameter as

$$
\mathcal{R}_{0}=\frac{\eta}{(\gamma+d+b)} \frac{\wedge}{b} \frac{(b+v)}{(b+v+\mu)} .
$$




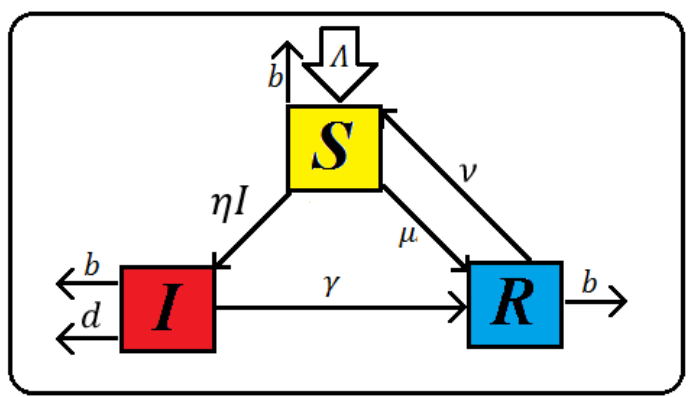

Figure 2. The movement of the individuals between compartments in the proposed model

It is clear that

$$
\mathcal{R}_{0}>0 \text {, }
$$

due to the inequalities in (7).

\section{Stability analysis}

Proposition 1 Let us consider the equations, $\frac{d^{\alpha}{ }_{1} S(t)}{d t^{\alpha}}=0, \frac{d^{\alpha_{2}} I(t)}{d t^{\alpha}{ }_{2}}=0, \frac{d^{\alpha} 3 R(t)}{d t^{\alpha} 3}=0$, for equilibrium points. The proposed model has two types of the equilibrium points. These are disease free equilibrium point $E_{0}\left(\frac{\Lambda}{b} \frac{(b+v)}{(b+v+\mu)}, 0, \frac{\Lambda}{b} \frac{\mu}{(b+v+\mu)}\right)$ and the endemic equilibrium $E_{1}\left(S^{*}, I^{*}, R^{*}\right)$ when

$$
\mathcal{R}_{0}>1 .
$$

In here, it is

$$
\left\{\begin{array}{l}
S^{*}=\frac{(\gamma+d+b)}{\eta}, \\
I^{*}=\frac{S^{*}\left(\mathcal{R}_{0}-1\right)(b+v+\mu)}{\left(\gamma+d+b+v \frac{d}{b}+v\right)}, \\
R^{*}=\left(\frac{S^{*}\left(\mathcal{R}_{0}-1\right)(b+v+\mu)}{\left(\gamma+d+b+v \frac{d}{b}+\gamma\right)}+\frac{S^{*}}{\gamma} \mu\right) \frac{\gamma}{(b+v)} .
\end{array}\right.
$$

Proposition 2 Considering the proposed model in (6), there are follows.

i. Let $\alpha_{1}=\alpha_{2}=\alpha_{3} \leq 1$. For CFOS, it is satisfied the followings:

a) If $\mathcal{R}_{0}<1$, the equilibrium point $E_{0}$, namely trivial disease-free equilibrium, is LAS.

b) If

$$
\left(\left(\eta I^{*}+(\mu+b)\right)+(\nu+b)\right)\left(\eta I^{*}\left((\nu+b)+\eta S^{*}\right)+b(\mu+v+b)\right)-\eta I^{*}\left((\nu+b) \eta S^{*}+\gamma v\right)>0,
$$

then the equilibrium point $E_{1}$, existing biologically meaning when $\mathcal{R}_{0}>1$, is LAS.

ii. For IFOS in system (6), where $\alpha_{1} \neq \alpha_{2} \neq \alpha_{3}<1$, it is satisfied the followings:

a) If $\mathcal{R}_{0}<1$ and all roots $\lambda_{i}$ for $i=1,2, \ldots, m\left(\alpha_{1}+\alpha_{3}\right)$ founded from the equation

$$
\lambda^{m\left(\alpha_{1}+\alpha_{3}\right)}+\lambda^{m \alpha_{1}}(v+b)+\lambda^{m \alpha_{3}}(\mu+b)+b(\mu+v+b)=0
$$

satisfy Routh-Hurwitz stability criteria [36] or the condition $\left|\arg \left(\lambda_{i}\right)\right|>\frac{1}{m} \frac{\pi}{2}$ [37] as seen inequalities (5), then the equilibrium point $E_{0}$ is LAS.

b) Let $\mathcal{R}_{0}>1$. If all roots $\lambda_{i}$ for $i=1,2, \ldots, m\left(\alpha_{1}+\alpha_{2}+\alpha_{3}\right)$ founded from the equation $\lambda^{m\left(\alpha_{1}+\alpha_{2}+\alpha_{3}\right)}+\lambda^{m\left(\alpha_{1}+\alpha_{2}\right)}(v+b)+\lambda^{m\left(\alpha_{2}+\alpha_{3}\right)}\left(\eta I^{*}+(\mu+b)\right)+\lambda^{m \alpha_{2}}\left(\eta I^{*}(v+b)+b(\mu+v+b)\right)$

$+\lambda^{m \alpha_{3}}(\gamma+d+b) \eta I^{*}+\eta I^{*}((\gamma+d+b)(v+b)+\gamma v)=0$ satisfy Routh-Hurwitz stability criteria or the condition $\left|\arg \left(\lambda_{i}\right)\right|>\frac{1}{m} \frac{\pi}{2}$, then the equilibrium point $E_{1}$ is LAS. 
Proof By the equations in (6), the Jacobian matrix evaluated at the equilibrium point $E_{i}(\bar{S}, \bar{I}, \bar{R})$ for $i=0,1$ is

$$
J\left(E_{i}\right)=\left(\begin{array}{ccc}
-(\eta \bar{I}+(\mu+b)) & -\eta \bar{S} & v \\
\eta \bar{I} & (\eta \bar{S}-(\gamma+d+b)) & 0 \\
\mu & \gamma & -(\nu+b)
\end{array}\right) .
$$

i. The system in (6) translates to CFOS, when $0<\alpha_{1}=\alpha_{2}=\alpha_{3} \leq 1$.

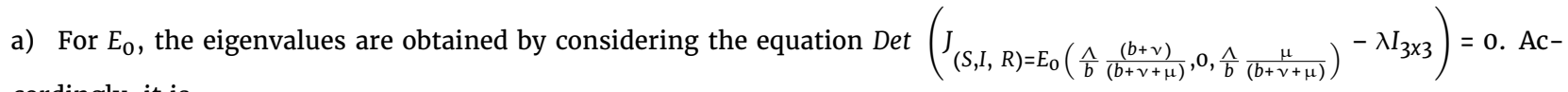
cordingly, it is

$$
\left(\lambda-(\gamma+d+b)\left(\mathcal{R}_{0}-1\right)\right)\left(\lambda^{2}+\lambda((\nu+b)+(\mu+b))+(\mu+v+b)\right)=0 .
$$

Therefore, the eigenvalues obtained from equation in (14) are determined as followings:

$$
\lambda_{1}=(\gamma+d+b)\left(\mathcal{R}_{0}-1\right)
$$

and $\lambda_{2}$ and $\lambda_{3}$ are found by solving the equation

$$
\lambda^{2}+\lambda((v+b)+(\mu+b))+(\mu+v+b) .
$$

It can be observed that $((\nu+b)+(\mu+b))>0$ and $(\mu+v+b)>0$, due to inequalities in (7). The LAS conditions for $E_{0}$ are provided for the eigenvalues $\lambda_{2}$ and $\lambda_{3}$. Thus, it is sufficient to examine the sign of $\lambda_{1}$. If

$$
\mathcal{R}_{0}<1
$$

then $\lambda_{1}$ is a negative real number due to inequalities (7). Routh-Hurwitz stability conditions are satisfied. In this case, $E_{0}$ is LAS.

b) Let $\mathcal{R}_{0}>1$. There is positive equilibrium point. Charasteristic equation obtained from $\operatorname{Det}\left(J_{(S, I, R)=E_{1}\left(S^{*}, I^{*}, R^{*}\right)}-\lambda I_{3 \times 3}\right)=0$ for the equilibrium point $E_{1}$ is founded as

$$
\lambda^{3}+a_{1} \lambda^{2}+a_{2} \lambda+a_{3}=0
$$

where

$$
a_{1}=\left(\eta I^{*}+(\mu+b)+(v+b)\right), a_{2}=\left(\eta I^{*}\left((v+b)+\eta S^{*}\right)+b(\mu+v+b)\right), a_{3}=\eta I^{*}\left((v+b) \eta S^{*}+\gamma_{I} v\right) .
$$

Let us consider that Routh-Hurwitz stability criteria. It is already clear that $a_{1}, a_{3}>0$ due to inequalities in (7) and (9). In addition, we have

$$
a_{1} a_{2}-a_{3}=\left(\left(\eta I^{*}+(\mu+b)\right)+(v+b)\right)\left(\eta I^{*}\left((v+b)+\eta S^{*}\right)+b(\mu+v+b)\right)-\eta I^{*}\left((v+b) \eta S^{*}+\gamma v\right) .
$$

If

$$
\left(\left(\eta I^{*}+(\mu+b)\right)+(\nu+b)\right)\left(\eta I^{*}\left((\nu+b)+\eta S^{*}\right)+b(\mu+v+b)\right)-\eta I^{*}\left((\nu+b) \eta S^{*}+\gamma v\right)>0
$$

then $a_{1} a_{2}-a_{3}>0$. Hence, $E_{1}$ is LAS when inequality in (20) is satisfied.

ii. In case of $0<\alpha_{1} \neq \alpha_{2} \neq \alpha_{3}<1$, we have IFOS of (6). In this sense, the determinant found by the equation

$$
\operatorname{det}\left(\operatorname{diag}\left(\lambda^{m \alpha_{1}}, \lambda^{m \alpha_{2}}, \lambda^{m \alpha_{3}}\right)-J\left(x_{1}, x_{2}, \ldots, x_{n}\right)=\left(\overline{x_{1}}, \overline{x_{2}}, \ldots, \overline{x_{n}}\right)\right)=0
$$

is

$$
\left|\begin{array}{ccc}
\lambda^{m \alpha_{1}}+(\eta \bar{I}+(\mu+b)) & -\eta \bar{S} & v \\
\eta \bar{I} & \lambda^{m \alpha_{2}}-(\eta \bar{S}-(\gamma+d+b)) & 0 \\
\mu & \gamma_{I} & \lambda^{m \alpha_{3}}+(\nu+b)
\end{array}\right|=0
$$

a) Firstly, if the determinant in (22) evaluates in the point $E_{0}\left(\frac{\Lambda}{b} \frac{(b+v)}{(b+v+\mu)}, 0, \frac{\mu}{(v+b)} \frac{\Lambda}{b} \frac{(b+v)}{(b+v+\mu)}\right)$ or $E_{0}\left(\frac{(\gamma+d+b)}{\eta} \mathcal{R}_{0}, 0, \frac{\mu}{(\gamma+b)} \frac{(\gamma+d+b)}{\eta} \mathcal{R}_{0}\right)$ with respect to (8), then it is achieved the equations:

$$
\lambda^{m \alpha_{2}}-(\gamma+d+b)\left(\mathcal{R}_{0}-1\right)=0
$$


and

$$
\left(\lambda^{m \alpha_{1}}+(\mu+b)\right)\left(\lambda^{m \alpha_{3}}+(v+b)\right)-\mu v=0 .
$$

These equations are examined as the followings: Taking into consideration the equation in (23), it is found that $\lambda^{m \alpha_{2}}=(\gamma+d+b)\left(\mathcal{R}_{0}-1\right)$. If

$$
\mathcal{R}_{0}<1
$$

then $\lambda^{m \alpha_{2}}$ is a negative real number due to inequalities in (7). Otherwise, at least one root of (23) would be a positive real num-

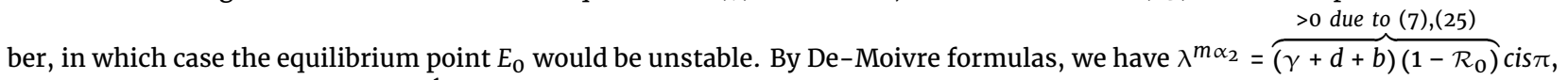
and so, $\lambda_{k}=\left[(\gamma+d+b)\left(1-\mathcal{R}_{0}\right)\right]^{\frac{1}{m \alpha_{2}}}$ cis $\left(\frac{\pi+2 k \pi}{m \alpha_{2}}\right)$ for $k=0,1,2, \ldots,\left(m \alpha_{2}-1\right)$, such that cis $\pi=\cos \pi+i \sin \pi, i=\sqrt{-1}$. Also, we have

$$
\left\{\begin{array}{l}
\left|\arg \left(\lambda_{0}\right)\right|=\frac{\pi}{m \alpha_{2}}, \\
\left|\arg \left(\lambda_{1}\right)\right|=\frac{3 \pi}{m \alpha_{2}}, \\
\vdots \\
\left|\arg \left(\lambda_{\left(m \alpha_{2}-1\right)}\right)\right|=\frac{\left(2 m \alpha_{2}-1\right) \pi}{m \alpha_{2}} .
\end{array}\right.
$$

Considering the conditions $|\arg (\lambda)|>\frac{\pi}{2 m}$ for the stability of the equilibrium point, the stability condition for $E_{0}$ is given as $\frac{\pi}{m \alpha_{2}}, \frac{3 \pi}{m \alpha_{2}}, \ldots, \frac{\left(2 m \alpha_{2}-1\right) \pi}{m \alpha_{2}}>\frac{\pi}{2 m}$, and so,

$$
\left\{\begin{array}{l}
\alpha_{2}<2, \\
\alpha_{2}<6, \\
\vdots \\
\alpha_{2}<2\left(2 m \alpha_{2}-1\right) .
\end{array}\right.
$$

Inequalities in (27) have been always provided since the derivative-orders $0<\alpha_{1}, \alpha_{2}, \alpha_{3} \leq 1$ in (6) are already satisfied. On the other hand, we have considered the equation (24)). If this equation is arranged, then

$$
\lambda^{m\left(\alpha_{1}+\alpha_{3}\right)}+\lambda^{m \alpha_{1}}(\nu+b)+\lambda^{m \alpha_{3}}(\mu+b)+b(\mu+\nu+b)=0
$$

is obtained. If the eigenvalues, which are the roots of equation (28), satisfy Routh-Hurwitz stability condition or the conditions $\left|\arg \left(\lambda_{i}\right)\right|>\frac{\pi}{2 m}$ for $i=1,2, \ldots, m\left(\alpha_{1}+\alpha_{3}\right)$, then $E_{0}$ is LAS.

b) Let $\mathcal{R}_{0}>1$. In this case, the equilibrium point $E_{1}$ emerges as positive definite. By calculating the determinant (22) at this equilibrium point, it is obtained the following characteristic equation

$$
\begin{aligned}
& \lambda^{m\left(\alpha_{1}+\alpha_{2}+\alpha_{3}\right)}+\lambda^{m\left(\alpha_{1}+\alpha_{2}\right)}(v+b)+\lambda^{m\left(\alpha_{2}+\alpha_{3}\right)}\left(\eta I^{*}+(\mu+b)\right)+ \\
& \lambda^{m \alpha_{2}}\left(\eta I^{*}(\nu+b)+b(\mu+\nu+b)\right)+ \\
& \lambda^{m \alpha_{3}}(\gamma+d+b) \eta I^{*}+\eta I^{*}((\gamma+d+b)(\nu+b)+\gamma v)=0 \text {. }
\end{aligned}
$$

When the signs of the terms of the last equation are examined according to Descartes' sign rule [38], it is clear that the equation does not have a positive real root. This does not disturb the stability of the equilibrium point. Therefore, if the eigenvalues $\lambda_{i}$ for $i=1,2, \ldots, m\left(\alpha_{1}+\alpha_{2}+\alpha_{3}\right)$, which are the roots of the equation (29), satisfy Routh-Hurwitz stability condition or the conditions $\left|\arg \left(\lambda_{i}\right)\right|>\frac{1}{m} \frac{\pi}{2}$, the equilibrium point $E_{1}$ is LAS.

Therefore, the proof is completed.

As a result, it can be reached to Table 3 .

Corollary 1 Let us consider Table 3. If $\mathcal{R}_{0}<1$ and some additional conditions are satisfied, then the equilibrium point $E_{0}$, always existing, is LAS. However, the equilibrium point $E_{1}$ biologically exists when $\mathcal{R}_{0}>1$. In this context, it can be said the followings:

i. In case the unexistence of $E_{1}, E_{0}$ can be a stable equilibrium point,

ii. In case the unstability of $E_{0}$, where $\mathcal{R}_{0}>1, E_{1}$ exists.

Therefore, these two points cannot be stable under the same conditions. 
Table 3. The existence conditions for the equilibrium points of system (6) and the stability conditions of these points according to different states of its derivative orders

\begin{tabular}{|c|c|c|c|}
\hline Equilibrium Point & $\begin{array}{l}\text { The exis- } \\
\text { tence con- } \\
\text { dition }\end{array}$ & Derivative-orders & Stability conditions \\
\hline$E_{0}\left(\begin{array}{c}\frac{\Lambda}{b} \frac{(b+v)}{(b+v+\mu)}, 0 \\
\frac{\Lambda}{b} \frac{\mu}{(b+v+\mu)}\end{array}\right.$ & Always & $\alpha_{1}=\alpha_{2}=\alpha_{3} \leq 1$ & If $\mathcal{R}_{0}<1$ \\
\hline$E_{0}\left(\begin{array}{c}\frac{\Lambda}{b} \frac{(b+v)}{(b+v+\mu)}, 0 \\
\frac{\Lambda}{b} \frac{\mu}{(b+v+\mu)}\end{array}\right.$ & Always & $\begin{array}{l}\alpha_{1} \neq \alpha_{2} \neq \alpha_{3} \\
\alpha_{1}, \alpha_{2}, \alpha_{3} \in(0,1)\end{array}$ & $\begin{array}{l}\text { If } \mathcal{R}_{0}<1 \text { and all roots } \lambda_{i} \text { for } i=1,2, \ldots, m\left(\alpha_{1}+\alpha_{3}\right) \text { founded } \\
\text { from the equation } \lambda^{m\left(\alpha_{1}+\alpha_{3}\right)}+\lambda^{m \alpha_{1}}(v+b)+\lambda^{m \alpha_{3}}(\mu+b)+ \\
b(\mu+v+b)=0 \text { satisfy Routh-Hurwitz stability criteria or the } \\
\text { condition }\left|\arg \left(\lambda_{i}\right)\right|>\frac{1}{m} \frac{\pi}{2} \text {. }\end{array}$ \\
\hline$E_{1}\left(S^{*}, I^{*}, R^{*}\right)$ & $\mathcal{R}_{0}>1$ & $\alpha_{1}=\alpha_{2}=\alpha_{3} \leq 1$ & $\begin{array}{l}\left(\left(\eta I^{*}+(\mu+b)\right)+(\nu+b)\right)\left(\eta I^{*}\left((\nu+b)+\eta S^{*}\right)+b(\mu+v+b)\right)- \\
\eta I^{*}\left((\nu+b) \eta S^{*}+\gamma v\right)>0\end{array}$ \\
\hline$E_{1}\left(S^{*}, I^{*}, R^{*}\right)$ & $\mathcal{R}_{0}>1$ & $\begin{array}{l}\alpha_{1} \neq \alpha_{2} \neq \alpha_{3} \\
\alpha_{1}, \alpha_{2}, \alpha_{3} \in(0,1)\end{array}$ & 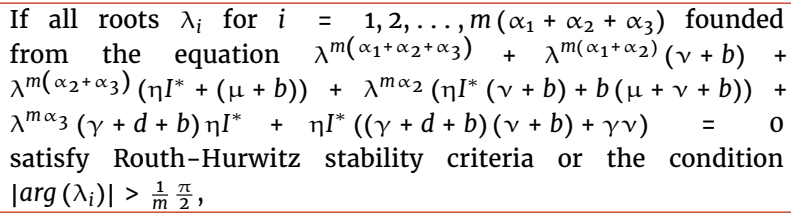 \\
\hline
\end{tabular}

\section{Numerical results}

To highlight the stability analysis results of this work using the proposed model for both CFOS and IFOS, two numerical examples are investigated. To do this, it is examined the behavior of the solutions of the model by valuing the parameters. It has been used Matlab R2012b. The parameter values are given in Table 4.

Table 4. The considered values of the parameters

\begin{tabular}{|c|c|c|c|}
\hline Parameter & Value $^{1}$ & Value $^{2}$ & Unit \\
\hline$\Lambda$ & 100 & 1000 & individuals \\
\hline $\mathrm{V}$ & 0.001 & 0.01 & day $^{-1}$ \\
\hline$\eta$ & 0.0001 & 0.0001 & day $^{-1}$ \\
\hline$\mu$ & 0.045 & 0.05 & day $^{-1}$ \\
\hline$b$ & 0.0032 & 0.15 & day $^{-1}$ \\
\hline$\gamma$ & 0.25 & 0.25 & day $^{-1}$ \\
\hline$d$ & 0.022 & 0.022 & day $^{-1}$ \\
\hline$\alpha_{1}$ & 0.9 & 0.8 & $\begin{array}{l}\text { Rational } \\
\text { number }\end{array}$ \\
\hline$\alpha_{2}$ & 0.9 & 0.6 & $\begin{array}{l}\text { Rational } \\
\text { number }\end{array}$ \\
\hline$\alpha_{3}$ & 0.9 & 0.4 & $\begin{array}{l}\text { Rational } \\
\text { number }\end{array}$ \\
\hline \multicolumn{4}{|c|}{ Value $^{1}$ is used in numerical study 1.} \\
\hline
\end{tabular}

\section{Numerical study 1}

Consider Value ${ }^{1}$ in Table 4 . It is found as $\mathcal{R}_{0} \approx 0.969$. This only means the existence of the equilibrium point $E_{0}(2668,0,28582)$. In addition, since $\mathcal{R}_{0}<1$, it is seen that the equilibrium point $E_{0}$ for CFOS $\left(\alpha_{1}=\alpha_{2}=\alpha_{3}=0.9\right)$ is stable according to Table 3 . This situation with initial conditions $\left[S_{0} I_{0} R_{0}\right]=\left[\begin{array}{lll}1000 & 1000 & 10000\end{array}\right]$ can be seen in Figures 3 and 4 .

\section{Numerical study 2}

When the values in Table 4 are used, the threshold parameter is found as $\mathcal{R}_{0} \approx 1.2$. Also, the trivial equilibrium point is $E_{0}(5079,0,1587)$. Since $\mathcal{R}_{0}>1$, the positive equilibrium point $E_{1}(4220,409,1958)$ exists and $E_{0}$ is an unstable point according to Table 3. Only the stability of $E_{1}$ can be examined.

Derivatives-orders are given as $\left[\begin{array}{lll}\alpha_{1} & \alpha_{2} & \alpha_{3}\end{array}\right]=\left[\begin{array}{lll}0.8 & 0.6 & 0.4\end{array}\right]$. Since $m$ is the least common multiple of the denominators of derivativeorders, it is 5. Equation (29) translates to

$$
\lambda^{9}+0.16 \lambda^{7}+0.2409 \lambda^{5}+0.038044 \lambda^{3}+0.0172598 \lambda^{2}+0.002863818=0 .
$$




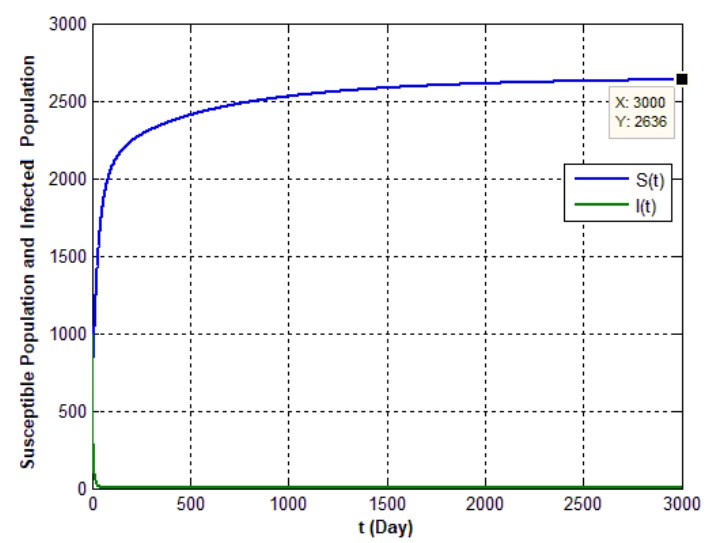

Figure 3. Time-dependent variation of susceptible and infectious populations for CFOS in (6)

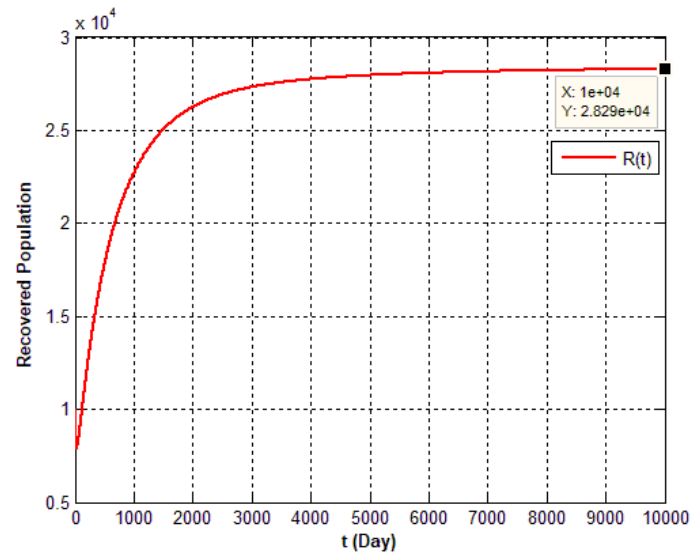

Figure 4. Time-dependent variation of the recovered population for CFOS in (6)

Roots of (30) are

$$
\begin{aligned}
\lambda_{1}= & -0.4990+0.5267 i, \\
\lambda_{2}= & -0.4990-0.5267 i, \\
\lambda_{3}= & 0.5039+0.4542 i, \\
\lambda_{4}= & 0.5039-0.4542 i, \\
& \lambda_{5}=-0.4042, \\
\lambda_{6}= & 0.2018+0.3738 i, \\
\lambda_{7}= & 0.2018-0.3738 i, \\
\lambda_{8}= & -0.0047+0.4025 i, \\
\lambda_{9}= & -0.0047-0.4025 i .
\end{aligned}
$$


Also, we have

$$
\begin{aligned}
\arg \lambda_{1} & =133.4530^{\circ}, \\
\arg \lambda_{2} & =226.5470^{\circ}, \\
\arg \lambda_{3} & =42.0305^{\circ}, \\
\arg \lambda_{4} & =317.9695^{\circ}, \\
\arg \lambda_{5} & =180^{\circ}, \\
\arg \lambda_{6} & =61.6371^{\circ}, \\
\arg \lambda_{7} & =298.3629^{\circ}, \\
\arg \lambda_{8} & =90.6690^{\circ}, \\
\lambda_{9} & =269.3310^{\circ} .
\end{aligned}
$$

Eigenvalues $\lambda_{i}$ for $i=1,2, \ldots, 9$ are greater than $\frac{\pi}{2 m}=18^{\circ}$. Therefore $E_{1}$ is LAS.

Let the initial conditions by $\left[S_{0} I_{0} R_{0}\right]=\left[\begin{array}{llll}10000 & 100 & 100\end{array}\right]$. In this case, the numerical simulation is obtained along the following Figures 5, 6 and 7.

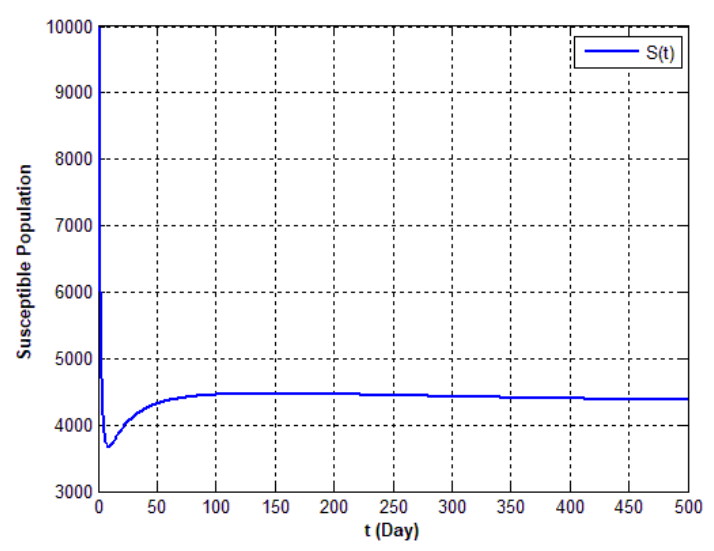

Figure 5. Time-dependent variation of susceptible population for IFOS in (6)

\section{Conclusions}

In this study, it is suggested the newly IFOS SIR model including the three time-dependent variables: susceptible, infected and recovered individuals in a population. This model proposed in system (6) is the form of nonlinear IFOS with the Caputo fractional derivative, accepted as rational numbers in the interval $(0,1]$. In this context, the general situation regarding the stability of proposed model was investigated. Considering the derivative-orders, a new perspective was presented to the literature.

The model has an infection-free equilibrium point $E_{0}\left(\frac{\Lambda}{b} \frac{(b+v)}{(b+v+\mu)}, 0, \frac{\Lambda}{b} \frac{\mu}{(b+v+\mu)}\right)$ and a positive equilibrium point

$E_{1}\left(S^{*}=\frac{(\gamma+d+b)}{\eta}, I^{*}=\frac{S^{*}\left(\mathcal{R}_{0}-1\right)(b+v+\mu)}{\left(\gamma+d+b+v \frac{d}{b}+\gamma\right)}, R^{*}=\left(\frac{S^{*}\left(\mathcal{R}_{0}-1\right)(b+v+\mu)}{\left(\gamma+d+b+v \frac{d}{b}+v\right)}+\frac{S^{*}}{\gamma} \mu\right) \frac{\gamma}{(b+v)}\right)$. For these equilibrium points, their existence were analyzed according to the threshold parameter $\mathcal{R}_{0}$ and their stability were examined according to both $\mathcal{R}_{0}$ and eigenvalues obtained from characteristic equation roots. These results about the stability analysis are summarized in Table 3. In general, the SIR models in literature trying to explain the infection progress in a population with respect to the only parameter $R_{0}$. According to qualitative analysis of our model, it was found followings: 


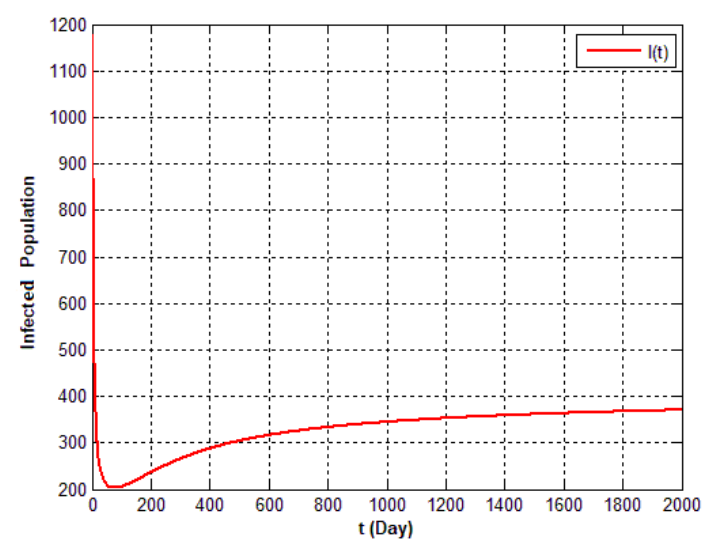

Figure 6. Time-dependent variation of infected population for IFOS in (6)

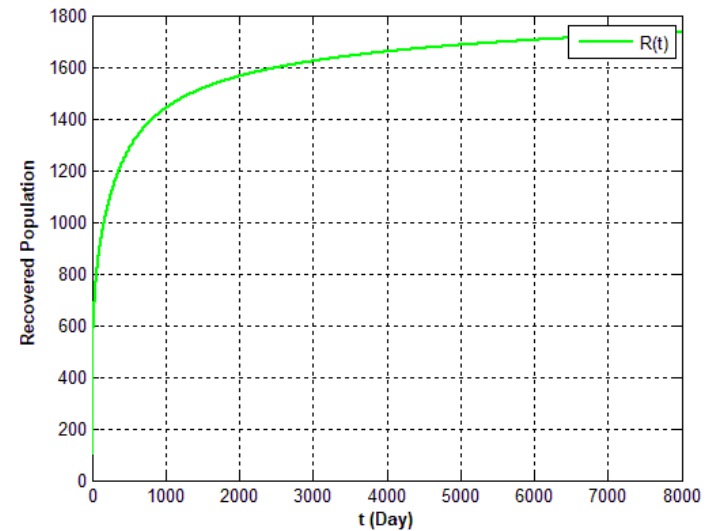

Figure 7. Time-dependent variation of recovered population for IFOS in (6)

i. Disease-free equilibrium point always exists and is LAS,

$$
\begin{cases}\text { If } R_{0}<1 & \text { in case of } \alpha_{1}=\alpha_{2}=\alpha_{3} \leq 1 . \\ \text { If } R_{0}<1 \text { and (28) meet conditions }\left|\arg \left(\lambda_{i}\right)\right|>\frac{\pi}{2 m} & \text { in other cases. }\end{cases}
$$

ii. Positive equilibrium point exists when $R_{0}>1$. This point is LAS,

$$
\begin{cases}\text { If } R_{0}>1 \text { (also the existence condition) } & \text { in case of } \alpha_{1}=\alpha_{2}=\alpha_{3} \leq 1 . \\ \text { If }(29) \text { meet conditions }\left|\arg \left(\lambda_{i}\right)\right|>\frac{\pi}{2 m} & \text { in other cases. }\end{cases}
$$

In numerical studies, the results of the qualitative analysis given in Table 3 are supported by graphics for the proposed SIR model. For this, the stability of $E_{0}$ for CFOS is shown in the first numerical study, while the stability of $E_{1}$ for IFOS is shown in the second numerical study.

\section{Declarations}

\section{Consent for publication}

Not applicable.

\section{Conflicts of interest}

The author declares that there is no known competing financial interests or personal relationships that could have appeared to influence the work reported in this paper.

\section{Funding}

The author declares that there is no funding source for the reported research. 


\section{Author's contributions}

The research was carried out by the author and he accepts that the contributions and responsibilities belong to the author.

\section{Acknowledgements}

Not applicable.

\section{References}

[1] Yavuz, M., Ozdemir, N. \& Baskonus, H. Solutions of partial differential equations using the fractional operator involving Mittag-Leffler kernel. The European Physical Journal Plus, 133(6), 1-11, (2018).

[2] Kashkynbayev, A. \& Rihan, F. Dynamics of Fractional-Order Epidemic Models with General Nonlinear Incidence Rate and Time-Delay. Mathematics, 9(15), 1829, (2021).

[3] Razminia, A., Majd, V. \& Baleanu, D. Chaotic incommensurate fractional order Rössler system: active control and synchronization. Advances In Difference Equations, 2011(1), 1-12, (2011).

[4] Ji, Y., Lu, J. \& Qiu, J. Stability of equilibrium points for incommensurate fractional-order nonlinear systems. 201635 th Chinese Control Conference (CCC), pp. 10453-10458, (2016,July).

[5] Daşbaşi, B. Stability analysis of the hiv model through incommensurate fractional-order nonlinear system. Chaos, Solitons \& Fractals, 137, 109870, (2020).

[6] Deng, W., Li, C. \& Guo, Q. Analysis of fractional differential equations with multi-orders. Fractals, 15(02), 173-182, (2007).

[7] Hamou, A.A., Azroul, E. \& Hammouch, Z. On dynamics of fractional incommensurate model of Covid-19 with nonlinear saturated incidence rate, medRxiv, (2021).

[8] Wang, Y. \& Li, T. Stability analysis of fractional-order nonlinear systems with delay. Mathematical Problems In Engineering, (2014).

[9] Petras, I. Stability of fractional-order systems with rational orders. ArXiv Preprint ArXiv:0811.4102, (2008).

[10] Rivero, M., Rogosin, S.V., Tenreiro Machado, J.A. \& Trujillo, J.J. Stability of fractional order systems. Mathematical Problems In Engineering, (2013).

[11] Brandibur, O., Garrappa, R. \& Kaslik, E. Stability of Systems of Fractional-Order Differential Equations with Caputo Derivatives. Mathematics, 9(8), 914, (2021).

[12] Lekdee, N., Sirisubtawee, S. \& Koonprasert, S. Bifurcations in a delayed fractional model of glucose-insulin interaction with incommensurate orders. Advances In Difference Equations, 2019(1), 1-22, (2019).

[13] Debbouche, N., Ouannas, A., Batiha, I.M. \& Grassi, G. Chaotic Dynamics in a Novel CovID-19 Pandemic Model Described by Commensurate and Incommensurate Fractional-Order Derivatives. (2021).

[14] Wang, X., Wang, Z. \& Xia, J. Stability and bifurcation control of a delayed fractional-order eco-epidemiological model with incommensurate orders. Journal of The Franklin Institute, 356(15), 8278-8295, (2019).

[15] Veeresha, P. A Numerical Approach to the Coupled Atmospheric Ocean Model Using a Fractional Operator.Mathematical Modelling and Numerical Simulation with Applications (MMNSA), 1(1), 1-10, (2021).

[16] Yavuz, M., \& Yaşkıran, B. Conformable Derivative Operator in Modelling Neuronal Dynamics. Applications \& Applied Mathematics, 13(2), 803-817, (2018).

[17] Yokuş, A. Construction of Different Types of Traveling Wave Solutions of the Relativistic Wave Equation Associated with the Schrödinger Equation. Mathematical Modelling and Numerical Simulation with Applications (MMNSA), 1(1), 24-31, (2021).

[18] Yavuz, M., \& Sene, N. Fundamental calculus of the fractional derivative defined with Rabotnov exponential kernel and application to nonlinear dispersive wave model. Journal of Ocean Engineering and Science, 6(2), 196-205, (2021).

[19] Hammouch, Z., Yavuz, M., \& Özdemir, N. Numerical Solutions and Synchronization of a Variable-Order Fractional Chaotic System. Mathematical Modelling and Numerical Simulation with Applications (MMNSA), 1(1), 11-23, (2021).

[20] Sene, N. Fractional diffusion equation described by the Atangana-Baleanu fractional derivative and its approximate solution. Journal of Fractional Calculus and Nonlinear Systems, 2(1), 60-75, (2021).

[21] Al-Mdallal, Q.M., Hajji, M.A., \& Abdeljawad, T. On the iterative methods for solving fractional initial value problems: new perspective. Journal of Fractional Calculus and Nonlinear Systems, 2(1), 76-81, (2021).

[22] Yavuz, M., Coşar, F. Ö., Günay, F., \& Özdemir, F. N. A new mathematical modeling of the COVID-19 pandemic including the vaccination campaign. Open Journal of Modelling and Simulation, 9(3), 299-321, (2021).

[23] Yavuz, M., Sulaiman, T.A., Yusuf, A. \& Abdeljawad, T. The Schrödinger-KdV equation of fractional order with Mittag-Leffler nonsingular kernel. Alexandria Engineering Journal, 60(2), 2715-2724, (2021).

[24] Nazir, G., Zeb, A., Shah, K., Saeed, T., Khan, R.A. \& Khan, S.I.U. Study of COVID-19 mathematical model of fractional order via modified Euler method. Alexandria Engineering Journal, 60(6), 5287-5296, (2021).

[25] Zarin, R., Ahmed, I., Kumam, P., Zeb, A. \& Din, A. Fractional modeling and optimal control analysis of rabies virus under the convex incidence rate. Results in Physics, 28, 104665, (2021).

[26] Alqudah, M.A., Abdeljawad, T., Zeb, A., Khan, I.U. \& Bozkurt, F. Effect of Weather on the Spread of COVID-19 Using Eigenspace Decomposition. Cmc-Computers Materials \& Continua, 3047-3063, (2021).

[27] Angstmann, C.N., Henry, B.I. \& McGann, A.V. A fractional-order infectivity and recovery SIR model. Fractal And Fractional, 1(1), 11, (2017).

[28] Liu, N., Fang, J., Deng, W. \& Sun, J.W. Stability analysis of a fractional-order SIS model on complex networks with linear treatment function. Advances In Difference Equations, 2019(1), 1-10, (2019).

[29] Alqahtani, R.T. Mathematical model of SIR epidemic system (COVID-19) with fractional derivative: stability and numerical analysis. Advances In Difference Equations, 2021(1), 1-16 (2021).

[30] Naik, P.A., Yavuz, M., Qureshi, S., Zu, J. \& Townley, S. Modeling and analysis of COVID-19 epidemics with treatment in 
fractional derivatives using real data from Pakistan. The European Physical Journal Plus, 135(10), 1-42, (2020).

[31] Tavares, D., Almeida, R. \& Torres, D.F. Caputo derivatives of fractional variable order: numerical approximations. Communications In Nonlinear Science And Numerical Simulation, 35, 69-87, (2016).

[32] Tavazoei, M.S. \& Haeri, M. Chaotic attractors in incommensurate fractional order systems. Physica D: Nonlinear Phenomena, 237(20), 2628-2637, (2008).

[33] Odibat, Z.M. Analytic study on linear systems of fractional differential equations. Computers \& Mathematics With Applications, 59(3), 1171-1183, (2010).

[34] Owolabi, K.M. Riemann-Liouville fractional derivative and application to model chaotic differential equations. Progress in Fractional Differentiation and Applications, 4, 99-110, (2018).

[35] Alshomrani, A.S., Ullah, M.Z. \& Baleanu, D. Caputo SIR model for COVID-19 under optimized fractional order. Advances In Difference Equations, 2021(1), 1-17, (2021).

[36] Daşbaşı, B. \& Öztürk, İ. Mathematical modelling of bacterial resistance to multiple antibiotics and immune system response. SpringerPlus, 5(1), 1-17, (2016).

[37] Daşbaşı, B. Stability analysis of mathematical model including pathogen-specific immune system response with fractionalorder differential equations. Computational And Mathematical Methods In Medicine, (2018).

[38] Daşbaşı, B. Çoklu Kesirli Mertebeden Diferansiyel Denklem Sistemlerinin Kalitatif Analizi, Analizdeki Bazi Özel Durumlar ve Uygulamasi: Av-Avci Modeli. Fen Bilimleri ve Matematik'te Akademik Araştırmalar (1. B., S. 127-157). Içinde Ankara: Gece Kitaplığı, (2018).

Mathematical Modelling and Numerical Simulation with Applications (MMNSA) (http://www.mmnsa.org)

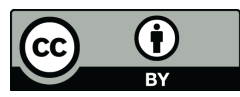

Copyright: ( 2021 by the authors. This work is licensed under a Creative Commons Attribution 4.0 (CC BY) International License. The authors retain ownership of the copyright for their article, but they allow anyone to download, reuse, reprint, modify, distribute, and/or copy articles in MMNSA, so long as the original authors and source are credited. To see the complete license contents, please visit (http://creativecommons.org/licenses/by/4.0/). 